\title{
Risk Factors of Patients with Prostate Cancer Upgrading for International Society of Urological Pathology Grade Group I After Radical Prostatectomy
} \author{
(1) Levent Türkeri ${ }^{5}$ \\ ${ }^{1}$ Marmara University Pendik Training and Research Hospital, Clinic of Urology, Istanbul, Turkey \\ 2 Marmara University Faculty of Medicine, Department of Urology, Istanbul, Turkey \\ ${ }_{3}^{3}$ Marmara University Faculty of Medicine, Department of Pathology, Istanbul, Turkey \\ ${ }^{4}$ Anatolian Health Center, Clinic of Urology, Istanbul, Turkey \\ 5 Mehmet Ali Aydınlar University Altunizade Hospital, Clinic of Urology, Istanbul, Turkey
}

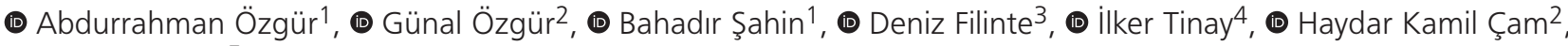

\begin{abstract}
Objective: This study aimed to determine the predictive factors for patients whose International Society of Urological Pathology (ISUP) score was upgraded in radical prostatectomy (RP) pathologies with a prostate biopsy pathology of ISUP grade group 1.

Materials and Methods: Among patients who underwent RP in our clinic within 10 years, 158 patients with prostate biopsy pathology of ISUP grade group 1 were examined retrospectively. Age, serum prostate-specific antigen (PSA) level, prostate biopsy ISUP grade group, number of cores taken in the prostate biopsy, number of tumor-positive cores, RP pathology ISUP grade group, and pathological stage were evaluated.

Results: The mean age ( \pm standard) of the 158 patients whose prostate biopsy pathology was ISUP grade group 1 were 64.07 ( \pm 6.6 ). ISUP group upgrading was detected in 47 patients $(29.7 \%)$. The mean PSA value of these patients was $10.6 \mathrm{ng} / \mathrm{mL}( \pm 6.9)$. The mean PSA value of the other 111 patients without ISUP group upgrading was $7.98 \mathrm{ng} / \mathrm{mL}( \pm 4.9)$. The serum PSA level was significantly higher in patients with upgraded ISUP in the RP pathology ( $p=0.02)$. The percentage of tumor-positive cores in the group with ISUP group upgrading (37\%) was significantly higher than that in the group without ISUP group upgrading (27\%) ( $p=0.01$ ). The detection rates of surgical margin positivity (42.6\% vs. $18 \%)$, capsule invasion ( $55.3 \%$ vs. $19.8 \%)$, and seminal vesicle invasion (23.6\% vs. $3.6 \%)$ were also significantly higher in the upgraded ISUP group after RP $(p<0.05)$.

Conclusion: The results of this trial suggest that active surveillance may not be an appropriate option for patients with biopsy ISUP grade group 1 with PSA level $>10 \mathrm{ng} / \mathrm{mL}$. Moreover, the presence of a higher number and percentage of tumor-positive cores constituted risks of ISUP group upgrading with concomitant poor pathological outcomes such as surgical margin positivity, capsule invasion, and seminal vesicle invasion.
\end{abstract}

Keywords: Prostate cancer, international society of urological pathology score, radical prostatectomy

\section{Introduction}

Prostate cancer is the second most common cancer among men after lung cancer and the $5^{\text {th }}$ cause of death after lung, liver, stomach, and colorectal cancers (1). With the use of prostate-specific antigen (PSA) as a marker from the late 1980 s onwards in prostate cancer screening, an increasing number of prostate cancer cases were detected (2). Mortality decreased due to increased prostate cancer diagnosis; however, it led to overdiagnosis and overtreatment in low-risk cases (3). The widespread use of PSA has led to the diagnosis of many asymptomatic cases as prostate cancer. Prostate cancer does not cause symptoms or death in a certain group of patients and does not affect the overall survival. The guidelines offer active surveillance (AS) as an alternative to curative treatment for lowrisk (PSA level $<10 \mathrm{ng} / \mathrm{mL}$, stage $<\mathrm{T} 2 \mathrm{a}$, Gleason score $<3+3$ ) and very low-risk (PSA level $<10 \mathrm{ng} / \mathrm{mL}$, stage $<1 \mathrm{c}$, Gleason score $<3+3$, PSA density $<0.15 \mathrm{ng} / \mathrm{mL},<3$ positive biopsy core count, $<50 \%$ core positivity) cases with clinically localized prostate cancer and life expectancy of over 10 years $(4,5)$. In intermediate-risk prostate cancer, AS can be recommended

Cite this article as: Özgür A, Özgür G, Şahin B, Filinte D, Tinay I, Çam HK, Türkeri L. Risk Factors of Patients with Prostate Cancer Upgrading for International Society of Urological Pathology Grade Group I After Radical Prostatectomy. Bull Urooncol 2022;21(1):10-13 
by discussing the risks with the patient with a low degree of recommendation (4). However, AS in intermediate-risk prostate cancer is controversial (6). Studies have shown that AS may be beneficial in selected patients with favorable intermediate-risk prostate cancer $(7,8)$.

A limited number of prospective randomized controlled studies are comparing AS with standard treatments (4). AS studies are generally cohort studies and have reported that patients demonstrated relatively good average survival and cancerspecific survival rates $(4,9)$. In a cohort study of patients with International Society of Urological Pathology (ISUP) grade group 1 followed up with AS, prostate cancer-specific 5-, 10-, and 15year cumulative metastasis and mortality rates were at $0.1 \%$ (10). However, in one-third of the patients, curative treatment is required depending on the upgrade of the disease, stage progression, or patient's preference (4).

AS aims to avoid unnecessary treatment in men with clinically localized prostate cancer that does not require emergency treatment and to provide cure at the most appropriate time in patients who need treatment during follow-up (11). Knowledge of the disease prognosis in the selection of clinical treatment is important. Since a limited amount of prostate tissue is sampled during biopsy, a risk of upgrading is inevitable after radical prostatectomy (RP). Therefore, it is important to reveal the risk factors for pathological upgrade in patients who received AS. At present, the best prognostic factors include the Gleason score, PSA level, and clinical tumor stage $(4,12)$.In this study, we aimed to determine the predictive factors for patients whose ISUP grade is upgraded in RP pathologies with prostate biopsy pathology of ISUP grade group 1.

\section{Materials and Methods}

Data of RP cases within 10 years at our urology department were retrospectively analyzed. Biopsy and RP pathologies were evaluated by the same pathology department. RP cases with a biopsy procedure and/or biopsy pathology evaluation performed at different centers were excluded. Moreover, RP cases with missing data for the PSA value, full biopsy pathology report, and RP pathology findings were excluded. Finally, RP cases with ISUP grade group 1 according to the prostate biopsy constituted the study group. Transrectal ultrasound (TRUS)guided biopsy was performed for histopathological diagnosis in all patients. ISUP grades were determined from Gleason scores recorded in the pathology report. As a result of staging, all patients have clinically localized prostate cancer. Age, serum PSA level, prostate biopsy ISUP grade group, number of cores taken in prostate biopsy, number of tumor-positive cores, RP pathology ISUP grade group, and pathological stage were evaluated. The patients were divided into two groups: group I with upgrading after RP and group 2 without upgrading based on the final pathology. These groups were compared to determine the risk factors of patients with ISUP group upgrade in RP pathologies. Approval for the study was obtained from Marmara University Clinical Research Ethical Committee (approval no: 09.2021.986, date: 03.09.2021).

\section{Statistical Analysis}

Data were analyzed using the independent sample t-test in the IBM Statistical Package for the Social Sciences Statics version 22. All these analyses used a significance level of $p<0.05$.

\section{Results}

Within 10 years, 289 patients with RP, whose full data were available, were evaluated. According to prostate biopsy pathologies, 158 patients had ISUP grade group 1, 61 had group 2, 20 had group 3, 28 had group 4, and 22 had group 5 . Conclusively, 158 patients with ISUP grade group 1 [mean age, 64.07 years $( \pm 6.6)$ ] according to the prostate biopsy were included in this study. Moreover, 47 patients (group $1 ; 29.7 \%$ ) with biopsy pathology ISUP grade group 1 were found to have an increase in the ISUP grade group based on the RP pathology. The mean PSA value of these patients was $10.6 \mathrm{ng} / \mathrm{mL}( \pm 6.9)$. Within all patients with prostate cancer, the RP pathology of 111 patients was reported as ISUP grade group 1. The mean PSA value of these patients was $7.98 \mathrm{ng} /$ $\mathrm{mL}( \pm 4.9)$. In the group comparison, the serum PSA level was

Table 1. Comparison of demographic, biochemical, and pathological findings based on prostate biopsy and radical prostatectomy of the two groups

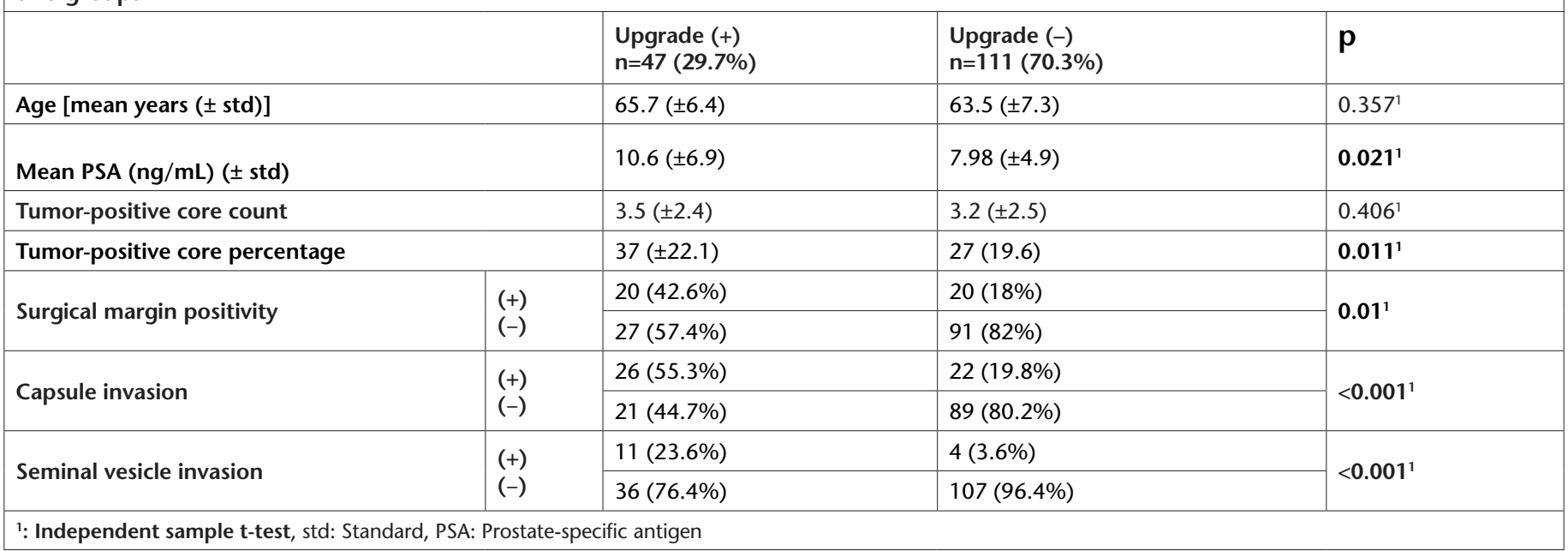


significantly higher in the group with ISUP group upgrading in the RP pathology $(\mathrm{p}=0.02)$.

The mean number of TRUS-guided prostate biopsy-positive cores in group 1 was higher than that in group 2 (Table 1). However, no significant difference was noted between the two groups ( $p=0.46$ ) based on the number of positive cores.

On the contrary, the percentage of tumor-positive cores in group $1(37 \%)$ was significantly higher than that in group 2 $(27 \%)(p=0.01)$. Similarly, the rates of surgical margin positivity $(42.6 \%$ vs. $18 \%)$, capsule invasion $(55.3 \%$ vs. $19.8 \%)$, and seminal vesicle invasion $(23.6 \%$ vs. $3.6 \%)$ were significantly remarkable in patients with ISUP grade group 1 in the final pathology $(p<0.05$, Table 1$)$.

\section{Discussion}

AS is recommended for localized low-risk prostate cancer cases to protect patients from the side effects of invasive curative treatments, such as radiotherapy and RP, and to maintain their quality of life (13). A study of intermediate-risk prostate cancer cases reported that survival was not different in the 10-year follow-up between AS and RP/RT; however, the incidence of disease progression and metastasis is lower in the RP/RT group (14). AS is controversial in the intermediate-risk group; thus, it is important to identify patients in the low-risk group. Findings in the initial biopsy pathology carry a vital guide in patient selection. However, a study including all ISUP grade groups showed that the discordance rate between prostate biopsy and RP pathologies was $35.7 \%$ (15). Another study reported a $36.3 \%$ upgrade in ISUP grade group of RP pathologies in patients who had ISUP grade group 1 in the prostate needle biopsy (16). In another study, the rate of Gleason score upgrading was $21.8 \%$ in low- and very low-risk cases after the final RP pathology (17). In a study of the AS group, Gleason score upgrading of $32 \%$ was observed in initial follow-up biopsies (18). In another study, Gleason score upgrading was observed in $13.8 \%$ of patients in the control biopsy (19). In the present study, about onethird of the patients (29.7\%) had ISUP group upgrading in the final RP pathology. This upgrading rate in the ISUP grade was comparable with the literature.

Determining the risk factors for possible upgrading in the ISUP evaluation of patients receiving AS is important. Studies have shown that a high PSA level, high PSA density, number of positive cores, percentage of positive cores, high clinical stage, advanced age, and high Gleason score are predictive risk factors for ISUP group upgrading $(17,19,20,21)$. According to our data analysis, the mean age of the group with ISUP group upgrading in the RP pathology was slightly higher without a significant difference $(p=0.357)$. Although $10 \mathrm{ng} / \mathrm{mL}$ is taken as the cutoff level for PSA value in AS in many studies, a study accepted higher PSA levels such as $15 \mathrm{ng} / \mathrm{mL}$ for AS (22). In our data, the PSA level was $10.6 \mathrm{ng} / \mathrm{mL}( \pm 6.9)$ in the group with ISUP group upgrading in RP pathologies and $7.98( \pm 4.9)$ in the group without upgrading. The PSA level was significantly higher in the group with ISUP group upgrading $(p=0.02)$. Consistent with the literature, PSA levels $<10 \mathrm{ng} / \mathrm{mL}$ in patients with prostate cancer of ISUP grade group 1 were more suitable for AS. When the PSA levels are $>10 \mathrm{ng} / \mathrm{mL}$, patients have a higher ISUP grade that could not be diagnosed by prostate biopsy. Therefore, these patients with PSA $>10 \mathrm{ng} / \mathrm{mL}$ should be informed about a higher risk of upgrading. Conclusively, these patients can actually be excluded from the AS program based on the higher change of upgrading after RP according to our results.

As the number and percentage of tumor-positive cores increase, the risk of ISUP group upgrading also increases. According to Akan et al. (23), the maximum percentage of core involvement was significantly higher in the upgraded group in the RP pathology. Our results were similar to the findings of this study. The percentage of tumor-positive cores was $37 \%( \pm 22.1)$, and it was significantly higher in the group with ISUP group upgrading $(p=0.01)$. When these data were evaluated, the risk of ISUP group upgrading was higher in patients with ISUP grade group 1 with $>3$ positive cores.

Although AS aims to avoid unnecessary treatment in men with clinically insignificant localized prostate cancer, it is very important to select the appropriate patient group. Failure to identify the low-risk group properly may cause irreversible problems. A patient with clinically significant prostate cancer will face the risk of disease progression during follow-up with AS. This disease progression will increase the morbidity and mortality of the patients. The rates of surgical margin positivity $(42.6 \%$ vs. $18 \%)$, capsule invasion (55.3\% vs. $19.8 \%)$, and seminal vesicle invasion ( $23.6 \%$ vs. $3.6 \%)$ were significantly higher in patients with ISUP group upgrading in RP pathologies than in patients without ISUP group upgrading $(p<0.05)$. Therefore, our results confirmed that upgraded cases after RP also had unfavorable pathological properties including positive surgical margin, capsule invasion, and seminal vesicle invasion.

\section{Study Limitations}

The major limitation of the current trial was the retrospective analysis of data. In addition, the number of patients was relatively limited. Randomized controlled prospective studies with larger number of patients are needed to determine definitive risk factors for ISUP group upgrading in patients with ISUP grade group 1 in the initial prostate biopsy and to identify patients with characteristics appropriate for AS.

\section{Conclusion}

Our results suggest that PSA $>10 \mathrm{ng} / \mathrm{mL}$ constitute a significant risk factor for upgrading after RP in patients with biopsy ISUP grade group I. Therefore, patients with a serum PSA level $>10 \mathrm{ng} / \mathrm{mL}$ and biopsy ISUP grade group 1 should be monitored very closely if they do not accept definitive treatment. An increased risk of upgrading after RP should be intensively discussed with these patients. Patients with biopsy ISUP grade group 1 with a higher number and/or percentage of tumor-positive cores also carry a remarkable risk of upgrading after RP. In conclusion, AS may not be an appropriate option in patients with PSA $>10 \mathrm{ng} / \mathrm{mL}$ and a higher number and percentage of tumor-positive cores due to the increased risk of ISUP group upgrading after RP. Moreover, these patients also demonstrated a higher risk of poor pathological outcomes including surgical margin positivity, capsule invasion, and seminal vesicle invasion. 


\section{Acknowledgements}

Publication: The results of the study were not published in full or in part in form of abstracts.

Contribution: There is not any contributors who may not be listed as authors.

Conflict of Interest: No conflict of interest was declared by the authors.

Financial Disclosure: The authors declared that this study received no financial support.

\section{Ethics}

Ethics Committee Approval: Approval for the study was obtained from Marmara University Clinical Research Ethical Committee (approval no: 09.2021.986, date: 03.09.2021).

Informed Consent: Retrospective study.

Peer-review: Externally peer-reviewed.

\section{Authorship Contributions}

Supervision: I.T., H.K.Ç., L.T., Concept: D.F., I.T., Design: A.Ö., G.Ö., B.Ş., D.F., I.T., Data Collection or Processing: A.Ö., G.Ö., B.Ş., Analysis or Interpretation: A.Ö., B.Ş., Literature Search: G.Ö., Writing: A.Ö., G.Ö.

\section{References}

1. Bray F, Ferlay J, Soerjomataram I, et al. Global cancer statistics 2018: GLOBOCAN estimates of incidence and mortality worldwide for 36 cancers in 185 countries. CA Cancer J Clin 2018;68:394-424.

2. Howlader N, Am N, Krapcho M, et al SEER Cancer Statistics Review, 1975-2010. National Cancer Instute, Seer Cancer, 2013.

3. Schröder FH, Hugosson J, Roobol MJ, et al. Prostate-cancer mortality at 11 years of follow-up. N Engl J Med 2012;366:981-990.

4. Mottet $\mathrm{N}$, van den Bergh RCN, Briers E, et al. EAU-EANM-ESTROESUR-SIOG Guidelines on Prostate Cancer-2020 Update. Part 1: Screening, Diagnosis, and Local Treatment with Curative Intent. Eur Urol 2021;79:243-262.

5. NCCN Clinical Practice Guidelines in Oncology Prostate Cancer Version 2, 2021.

6. Loeb S, Folkvaljon Y, Bratt O, et al. Defining Intermediate Risk Prostate Cancer Suitable for Active Surveillance. J Urol 2019;201:292-299.

7. Klotz L. Active surveillance in intermediate-risk prostate cancer. BJU Int 2020;125:346-354.

8. Raldow AC, Zhang $\mathrm{D}, \mathrm{Chen} \mathrm{MH}$, et al. Risk Group and Death From Prostate Cancer: Implications for Active Surveillance in Men With Favorable Intermediate-Risk Prostate Cancer. JAMA Oncol 2015;1:334-340.
9. Klotz L, Vesprini D, Sethukavalan P, et al. Long-term follow-up of a large active surveillance cohort of patients with prostate cancer. J Clin Oncol 2015;33:272-277.

10. Tosoian JJ, Mamawala M, Epstein Jl, et al. Active Surveillance of Grade Group 1 Prostate Cancer: Long-term Outcomes from a Large Prospective Cohort. Eur Urol 2020;77:675-682.

11. Bruinsma SM, Roobol MJ, Carroll PR, et al. Expert consensus document: Semantics in active surveillance for men with localized prostate cancer - results of a modified Delphi consensus procedure. Nat Rev Urol 2017;14:312-322.

12. Lee H, Lee M, Byun SS, et al. Evaluation of Prostate Cancer Stage Groups Updated in the 8th Edition of the American Joint Committee on Cancer Tumor-Node-Metastasis Staging Manual. Clin Genitourin Cancer 2019;17:221-226.

13. Klotz L. Active surveillance for low-risk prostate cancer. Curr Opin Urol 2017;27:225-230.

14. Hamdy FC, Donovan JL, Lane JA, et al. 10-Year Outcomes after Monitoring, Surgery, or Radiotherapy for Localized Prostate Cancer. N Engl J Med 2016;375:1415-1424.

15. Öztürk E, Yıkılmaz TN. Gleason Score Correlation Between Prostate Biopsy and Radical Prostatectomy Specimens. Bull Urooncol 2018;17:1-4.

16. Epstein JI, Feng Z, Trock BJ, Pierorazio PM. Upgrading and downgrading of prostate cancer from biopsy to radical prostatectomy: incidence and predictive factors using the modified Gleason grading system and factoring in tertiary grades. Eur Urol 2012;61:1019-1024.

17. Tosoian JJ, JohnBull E, Trock BJ, et al. Pathological outcomes in men with low risk and very low risk prostate cancer: implications on the practice of active surveillance. J Urol 2013;190:1218-1222.

18. Osses DF, Drost FH, Verbeek JFM, et al. Prostate cancer upgrading with serial prostate magnetic resonance imaging and repeat biopsy in men on active surveillance: are confirmatory biopsies still necessary? BJU Int 2020;126:124-132.

19. Tosoian JJ, Trock BJ, Landis P, et al. Active surveillance program for prostate cancer: an update of the Johns Hopkins experience. J Clin Oncol 2011;29:2185-2190.

20. Cary KC, Cowan JE, Sanford M, et al. Predictors of pathologic progression on biopsy among men on active surveillance for localized prostate cancer: the value of the pattern of surveillance biopsies. Eur Urol 2014;66:337-342.

21. Haberal HB, Artykov M, Hazir B, et al. Predictors of ISUP score upgrade in patients with low-risk prostate cancer. Tumori 2021;107:254-260.

22. Selvadurai ED, Singhera $M$, Thomas K, et al. Medium-term outcomes of active surveillance for localised prostate cancer. Eur Urol 2013;64:981-987.

23. Akan S, Ediz C, Temel MC, et al. Correlation of the Grade Group of Prostate Cancer according to the International Society of Urological Pathology (Isup) 2014 Classification between Prostate Biopsy and Radical Prostatectomy Specimens. Cancer Invest 2021;39:521-528. 